\title{
EXPRESSION OF A NOVEL GENE ENCODING PROTEASE INHIBITOR FROM METAGENOME OF SPONGE IN VIETNAM
}

\author{
Tran Thi Hong ${ }^{1,3^{*}}$, Ton That Huu Dat ${ }^{1}$, Nguyen Phuong Hoa ${ }^{1}$, \\ Pham Viet Cuong ${ }^{1}$, Nguyen Thi Kim Cuc ${ }^{2}$ \\ ${ }^{1}$ Mien Trung Institute for Scientific Research, VAST \\ ${ }^{2}$ Institute of Marine Biochemistry, VAST \\ ${ }^{3}$ Graduate University of Science and Technology, VAST
}

\begin{abstract}
Marine sponge is known as a "gold mine" of natural products from marine environment. Many novel bioactive compounds have been isolated from marine sponges and sponge-associated microorganisms such as antibiotics, anti-cancer compounds, protease inhibitors, etc. In this study, we selected a gene encoding protease inhibitor from metagenome of a sponge collected in Quang Tri to express in Escherichia coli (E. coli) BL21(DE3). The gene PI-DN9 encoding protease inhibitor $(1.3 \mathrm{~kb})$ was cut off cloning vector pUC57/PI-DN9 containing gene PI-DN9 and inserted into expression vector pET-32a(+), the recombinant vector pET-32a(+)/PI-DN9 then was transformed and expressed in the E. coli strain BL21(DE3). Results showed that recombinant protein $(50 \mathrm{kDa})$ was expressed successfully at $25^{\circ} \mathrm{C}, 1 \mathrm{mM}$ of IPTG in 5 hours. The recombinant protein was purified using Ni-NTA affinity chromatography column. Western blot assay and bioactive assay showed good activity of the purified protein.
\end{abstract}

Keywords: Escherichia coli, expression vector, protease inhibitor, recombinant protein, sponge.

Citation: Tran Thi Hong, Ton That Huu Dat, Nguyen Phuong Hoa, Pham Viet Cuong, Nguyen Thi Kim Cuc, 2018. Expression of a novel gene encoding protease inhibitor from metagenome of sponge in Vietnam. Academia Journal of Biology, 40(4): 77-84. https://doi.org/10.15625/2615-9023/v40n4.12759.

*Corresponding author email: tranhongtrn@gmail.com

Received 13 July 2018, accepted December 2018

\section{INTRODUCTION}

Proteases are important enzymes in host cells and responsible for many physiological functions, such as zymogene activation, coagulation, fibroblast fibrinolysis, hormone and bioactive peptide release, etc. However, the loss of control of proteolysis can cause cancer and cardiovascular diseases, inflammation, neuro degeneration as well as bacterial, viral and parasitic diseases. Nowadays, protease inhibitors are often used for treatment of diseases related to proteases. Therefore, discovery of novel protease inhibitors is still a special interest of scientists (Barrett et al., 1998; Agbowuro et al., 2017).
Metagenomics is new approach that allowsstudying genetic materials recovered directly from environment samples. To date, metagenomics has been used for finding novel bioactive compounds as well as investigating diversity of microorganisms from different environments. Particularly, studies focus on discovery of genes encoding potential bioactive compounds for bio-pharmaceutical field from marine microorganisms. Many protease inhibiors and other bioactive second metabolites have found from metagenome database (Baharum et al., 2010; Culligan et al., 2014; He et al., 2013). 
In this study, we selected a novel gene encoding protease inhibitor from metagenome of marine sponge QT collected in Quang Tri and expressed in the Escherichia coli (E. coli) strain BL21(DE3) for finding and mining novel protease inhibitor.

\section{MATERIALS AND METHODS}

Cloning vector pUC57 (Genscript, USA) inserted gene PI-DN9 from metagenome of the sponge QT; expression vector pET32a(+); strain E. coli Top10F'; strain E. coli BL21(DE3) (Invitrogen, USA); restriction enzyme EcoRI, NotI and DNA marker (Fermentas, USA); protein markers (Novagen, Netherlands; Sigma-Aldrich, USA, iNtRON Biotechnology, Korea; Affymetrix, USA); Thrombin (Novagen, The Netherlands); Skimmed milked (Difco, USA); trypsin, achymotrypsin, thermolysin, IPTG, NBT (Sigma-Aldrich, USA).

\section{Construction of recombinant vector pET-32a(+)/PI-DN9}

Metagenome of the sponge QT was sequenced and annotated using databases, such as CAZy (Cantarel et al., 2009), GO (Ashbumer et al., 2006), COG, SWISSPROT, KEGG (Tatusov et al., 2001) and tools such as dbCAN (Yin et al., 2112), Prodigal (Hyatt et al., 2010) and MetageneMark (Zhu et al., 2010) by BaseClear (Netherlands). The contig 00046 (prokka 10704) having similarity 50\% with genes encoding protease inhibitor on GeneBank was selected for protein expression. The sequence of contig 00046 was designed to contain two restriction enzymes EcoRI and NotI (PI-DN9) and inserted in cloning vector pUC57. The gene PI-DN9 was cut from cloning vector pUC57/PI-DN9 using enzymes EcoRI và NotI. The expression vector pET-32a(+) was also cut using two above enzymes. Finally, the gene PI-DN9 was inserted into expression vector $\mathrm{pET}-32 \mathrm{a}(+)$ in order to create recombinant vector pET32a(+)/PI-DN9.

\section{Expression of the gene PI-DN9 in E. coli BL21(DE3)}

The recombinant vector pET-32a(+)/PIDN9 was transformed into E.coli BL21(DE3) competent cells by the heat shock method. The strain E.coli BL21(DE3) containing the recombinant vector was incubated for overnight in LB medium supplemented with $50 \mu \mathrm{g} / \mathrm{mL}$ of ampicillin (LBamp) at $37^{\circ} \mathrm{C}$ and shaked at $200 \mathrm{rpm}$. Culture broth was transferred into fresh Lbamp medium and incubated in the same above conditions until $\mathrm{OD}_{600}=0.6$, then added $1 \mathrm{mM}$ of Isopropylthio- $\beta$-galactosida (IPTG) into culture broth and expressed at $25^{\circ} \mathrm{C}$ in 5 hours. The expressed protein was checked on $12.6 \%$ SDS-PAGE gel.

\section{Western blot assay}

In this study, Western blot assay was used to detect protease inhibitory protein. Protein was separated on $12.6 \%$ SDS-PAGE gel then transferred onto nitrocellulose or polyvinylidenedifluoride (PVDF) membrance by liquid blotting system (Invitrogen). The membrance was blocked with 5\% milk in TBS for overnight and was incubated with antibody anti-TRx at $4{ }^{\circ} \mathrm{C}(1: 1000)$ for 2 hours. The membrance then was placed in TBS buffer and washed for 10 minutes. Next, the membrance was incubated with the secondary antibody solution for 1 hours at room temperature, then was placed in TBS and washed for 10 minutes. This step was repeated twice with fresh buffer. Finally, the NBT substrate was added onto membrance to visualize the protein activity.

\section{Determination of protein in supernatant and pellet}

Culture broth was centrifuged at $5000 \mathrm{rpm}$ for 5 minutes and removed supernatant. The pellet was resuspended in TE buffer $(20 \mathrm{mM}$ Tris, $10 \mathrm{mM}$ EDTA, $0.05 \mathrm{mM}$ PMF) and incubated at $(-) 80^{\circ} \mathrm{C}$ for 1 hour. The pellet was thawed at $50^{\circ} \mathrm{C}$ in 30 minutes and 
disrupted by ultrasound. The solution was centrifuged at $13.000 \mathrm{rpm}$ for 15 minutes. The supernatant was collected, and the pellet was resuspended again in TE buffer. Finally, SDS loading bufferwas added into culture broth before centrifugating. After centrifugating, supernatant and the pellet were deraturated at $100^{\circ} \mathrm{C}$ for 10 minutes. The expression protein was checked on $12.6 \%$ SDS-PAGE gel.

\section{Purification of protein PI-DN9}

The recombinant protein was purified by Invitrogen ${ }^{\mathrm{TM}}$ Novex $^{\mathrm{TM}}$ Ni-NTA purification system according to manufacture's protocol. Then, the purified protein was then cut off TRx-His tagusing Thrombin Kit (Novagen, The Netherlands).

\section{Primary screening of protease inhibitory activity of protein}

Protease inhibitory activity of the recombinant protein was performed in skimmed milk agar plate $(0.8 \%$ agar, $1 \%$ skimmed milked) with wells made in centre and periphery at a distance of $1 \mathrm{~cm}$ from the central well. Twenty microlitres of proteases (trypsin, or a-chymotrypsin, or thermolysin at $0.5 \mathrm{mg} / \mathrm{ml}$ ) were pipetted in the central well. The recombinant protein was added in one of the peripheral wells and sterile distilled water (negative control) in the other. The plate was incubated at $37^{\circ} \mathrm{C}$. The inhibitory activity was detected by absence of clearance zone around the well containing the inhibitor (Sapna, 2013).

\section{Protease inhibitory assay of the recombinant protein}

The recombinant protein was added to a mixture of chloride, phosphate buffer and protease solutions (trypsin, or achymotrypsin, or thermolysin). After adjusting the $\mathrm{pH}$ to 7.5 , BAPNA (N-abenzoyl-DL-arginine-p-nitroanilide) was added into the mixture and incubated at $37^{\circ} \mathrm{C}$ for 20 minutes. Next, 5\% TCA was added into the solution and incubated at room temperature for 20 minutes. Finally, the solution was filtered with Whatman no. 1 filter paper and the absorbance was measured at $280 \mathrm{~nm}$. The inhibiting activity of protease inhibitor was calculated following formula:

Inhibiting activity $(\%)=\mathrm{C}-\mathrm{T} / \mathrm{C} \times 100$

$\mathrm{C}$ was absorbance value of blank sample and $\mathrm{T}$ was absorbance value of samples with protease inhibitor (Karthik et al., 2014).

\section{RESULTS AND DISCUSSION}

\section{Construction of recombinant vector pET- 32a(+)/PI-DN9}

The gene PI-DN9 was cut from plasmid pUC57/PI-DN9 using restriction enzymes EcoRI and NotI. The vector pET-32a(+) was also digested with EcoRI and NotI (Fig. 1A). The products were then purified and checked on $1 \%$ agarose gel (Fig. 1B). Agarose gel analysis showed that there was a band with size of $6 \mathrm{~kb}$ at lane 1 and lane 3 which was similar to the size of vector pET-32a(+). The band with size of $2.7 \mathrm{~kb}$ at lane 2 was similar to the size of vector pUC57 and the band in lane 4 with the size of $1.3 \mathrm{~kb}$ was similar to the size of gene PI-DN9.

The gene PI-DN9 was inserted into vector pET-32a (+) using T4 ligase, then transformed into strain E. coli Top10F' competent cells and incubated in LB medium containing $1 \mathrm{mM}$ ampicillin. Some colonies were randomly picked for plasmid extraction and then digested with EcoRI và NotI. The cutting products were checked on $1 \%$ agarose gel (Fig. 2).

The results showed that, the presenced bands with $1.3 \mathrm{~kb}$ in size at lane 1 and 2 (Fig. 2) were similar to the size of the gene PI-DN9 $(1.3 \mathrm{~kb})$. Besides, we detected other bands with $6 \mathrm{~kb}$ in size at lane 1 and 2 were similar to the size of the vector pET-32a(+) without gene PI-DN9 at lane C. This result indicated that gene PI-DN9 may be inserted successfully into vector pET-32a(+). To 
confirm this, we sequenced recombinant plasmid and result showed that the sequenced gene was similar $100 \%$ with gene PI-DN9

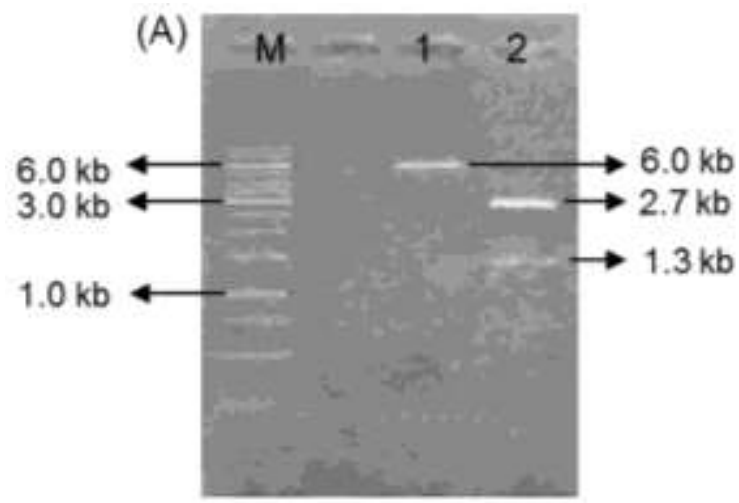

(data not shown). Therefore, the expression vector pET-32a(+)/PI-DN9 was successfully constructed.

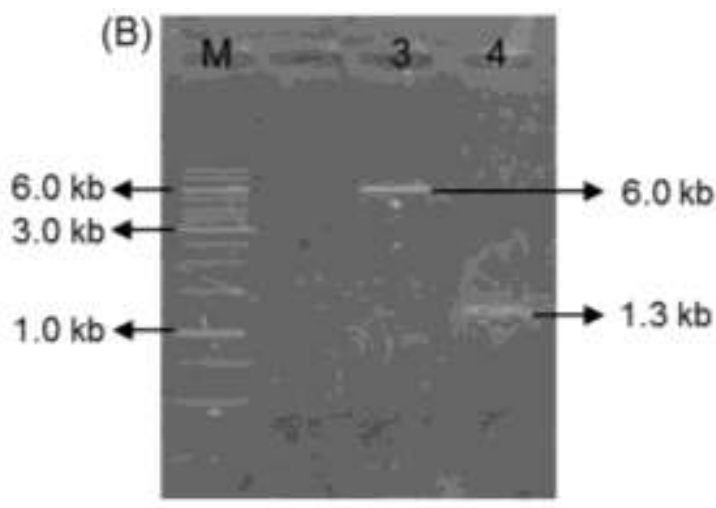

Figure 1. (A) Agarose gel analysis of vector pET-32a(+) and vector pUC57/PI-DN9 cut with EcoRI andNotI, (B) Purified products. Lane M: Maker $1 \mathrm{~kb}$ (Thermo), Lane 1(A): opened vector pET-32a (+) after cut with EcoRI andNotI, Lane 3: purified vector pET-32a(+), Lane 2(A): opened vector pUC57/PI-DN9 cut with EcoRI and NotI, Lane 4: purified gene PI-DN9

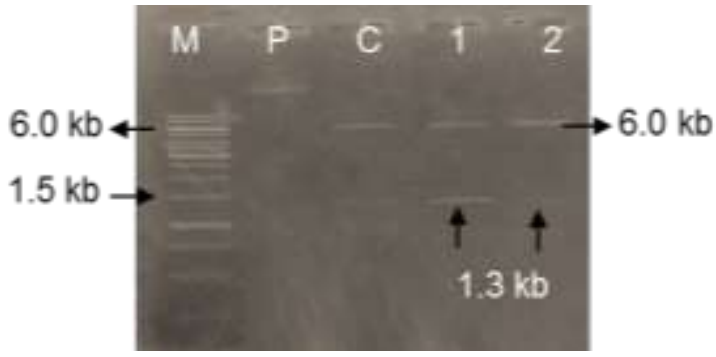

Figure 2. Agarose gel analysis of recombinant plasmid cut with EcoRI and NotI. lane $\mathrm{M}$ : marker $1 \mathrm{~kb}$ (Thermo); lane 1: plasmid pET-32a(+)/PI-DN9; lane 2, 3, 4: plasmid pET-32a(+)/PI-DN9 was cut by enzymes EcoRI/NotI; lane 5: vector pET-32a(+)

\section{Expression of recombinant protein in E. coli BL21(DE3)}

The recombinant vector pET-32a(+)/PIDN9 was transformed into $E$. coli BL21(DE3) competent cells and incubated in LB medium with $1 \mathrm{mM}$ (amp). The presence of white colonies on plate indicated the recombinant vector may be transformed successfully in E. coli BL21(DE3). To confirm this, we picked randomly 3 colonies and expressed recombinant protein as described in the methods section (Fig. 3).

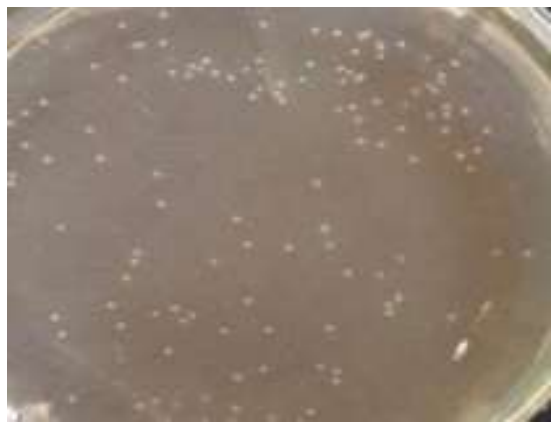

Figure 3. Colonies of E. coli BL21(DE3) containing recombinant vector pET32a(+)/PI-DN9 on LBamp medium

Expressed recombinant protein was checked on SDS-PAGE gel and stained with Coomassie (Fig. 4A). The result showed that colonies induced with IPTG appeared an extra band with the size of $64 \mathrm{kDa}$, which is similar with the size of protein PI-DN9 attached TRx-His tag (Fig. 4A: lane 3,5,7). Lane 1 (only vector pET-32a(+) and lane 2, 4, 6 (colonies were not induced with IPTG) did not have this extra band. To confirm the expression abilities of recombinant protein, we selected one clone and performed Western blot assay with the specific anti-TRx antibody. 
Western blot analysis showed a band with the size of $64 \mathrm{kDa}$, which was similar to the result of SDS-PAGE analysis. Therefore, we confirmed that the recombinant protein was expressed successfully.

In order to determine the fraction of expression protein, the recombinant protein in the supernatant and pellet fractions were denatured and checked on $12.6 \%$ SDS-PAGE gel. The SDS-PAGE analysis showed that the recombinant protein was expressed mainly in supernatant fraction (Fig. 4C, lane T).

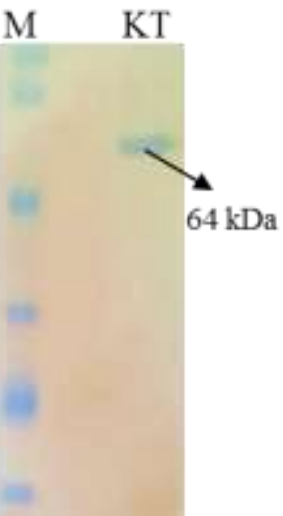

Figure 4. Analysis of protein expression on SDS-PAGE gel: (A) Protein expression PI-DN9 at $25^{\circ} \mathrm{C}$ and $1 \mathrm{mM}$ of IPTG (Lane 1: clone without gen PI-DN9, Lane 2, 4, 6: clones containing Pet32a(+)/PI-DN9 without induction with IPTG, Lane 3, 5, 7: clones containing pET-32a(+)/PI-DN9 induced with IPTG, Lane M: protein maker, (B) Western blot reaction of protein PI-DN9 with TRx (Land KT), (C) Protein expression in cultrure broth (TC), in pellet (C), in supernatant (T)

Purification of expressed protein and determination of its protease inhibitory activity

The vector pET-32a(+) contained the sequence of Hig-tag was used for protein expression, thereby the recombinant protein was purified by affinity chromatographic method using Ni-NTA purification system, then the purified protein fractions were checked on $12.6 \%$ SDS-PAGE gel. The result showed that, the recombinant protein PI-DN9 was in stage 1 and 2 . However, amount of purified protein in stage 1 was higher than in stage 2 (Fig. 5A).

In order to test protease inhibitory activity of the expressed protein, the sequence of TRx$H i s$ tag was removed from the purified protein using thrombin. The SDS-PAGE analysis of the protein treated with thrombin revealed the presence of two bands: one band had size of $50 \mathrm{kDa}$ (size of protein PI-DN9) and one band with size of $14 \mathrm{kDa}$ (size of TRx-His tag)(Fig.
5B). Therefore, we removed successfully TRx-His tag from the expressed protein.

Activity assay results of expressed protein against proteases (trypsin, a-chymotrypsin, thermolysin) using skimmed milked plate showed that protein PI-DN9 displayed inhibitory activity against trypsin (Fig. 6), but did not inhibit against a-chymotrypsin and thermolysin. Protease inhibitory assay indicated that inhibitory activity of protein against trypsin reached $83 \%$.

There are some studies focused on discovery of protease inhibitory proteins from microorganisms associated with marine sponge; however, most of them use cultivation-dependent approaches (Wahyudi et al., 2010; Tabares et al., 2011; PimentelElardo et al., 2011; Ramadan et al., 2012). With rapid development of advance sequencing technology, scientists begin discovering and mining novel natural products 
based on metagenomics instead of traditional cultivation. For example, Jiang et al. (2011) cloned successfully a novel protease inhibition gene against serine (Spi1C) based on metagenome of marine microorganisms.

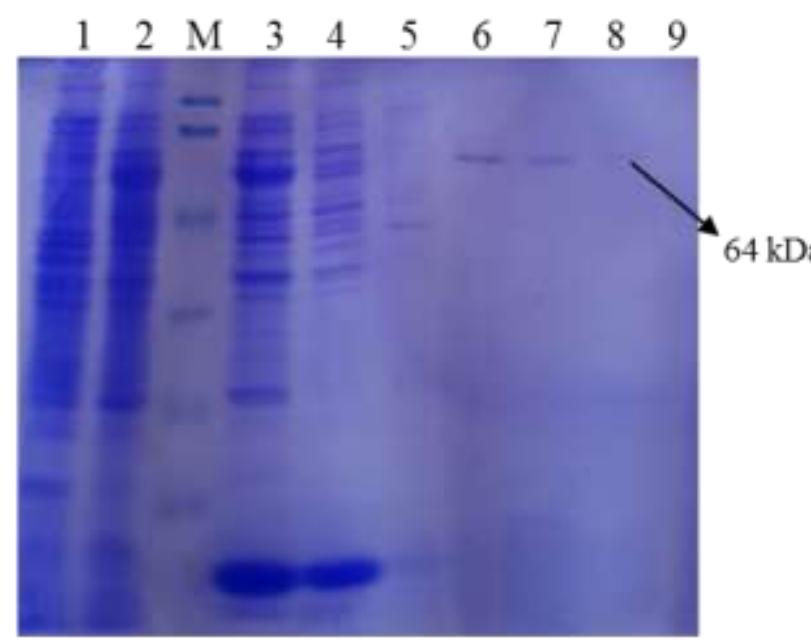

The gene Spi1C had ORF with 642 bp and encoded for 214 amino acid with estimated size of $28.7 \mathrm{kDa}$. The protein SpilC displayed inhibitory activity against serine proteases such as $\alpha$-chymotrypsin và trypsin.

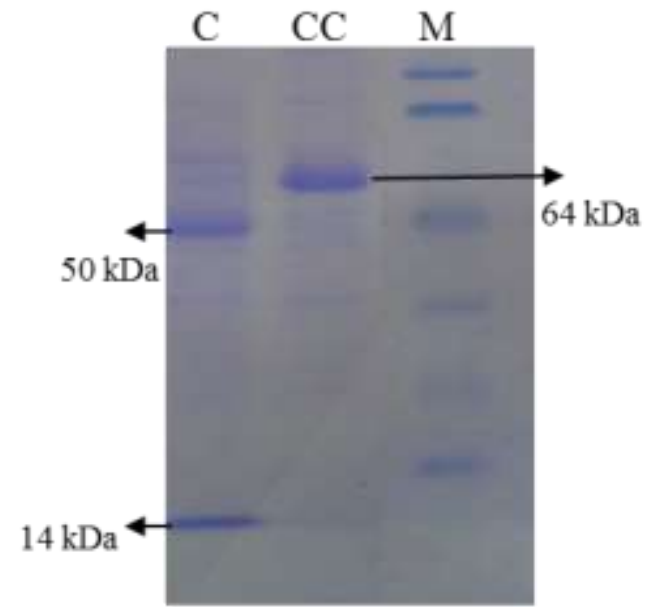

Figure 5. Analysis of the expressed protein after purifying on SDS-PAGE gel: (A) Fractions of expressed protein after purifying with Ni-NTA column (Lane M: protein marker (Bio-basic), Lane 1: protein without induced with IPTG, Lane 2: protein induced with IPTG, Lane 3: protein before purifying, Lane 4: protein after purifying with Ni-NTA column, Lane5: protein after column treated with imidazole $100 \mathrm{mM}$, Lane 6-10: proteine fractions after treating column with imidazole 250mM), (B) Protein expression after cut TRx-His (Land C), before cutting TRx-His (Lane CC) and protein maker of Bio-basic (M)
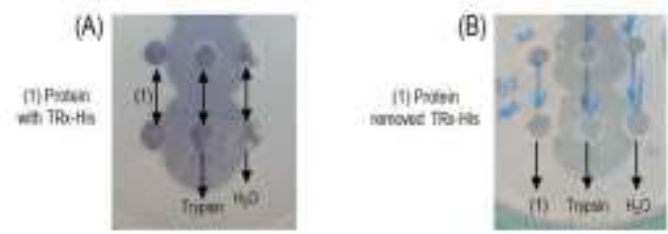

Figure 6. Protease inhibitor assay on plate

(A) protein with TRx-His tag, (B) protein removed TRx-His tag

\section{CONCLUSION}

A novel gene encoding protease inhibitor PI-DN9 from metagenome of a sponge in Quang Tri was expressed successfully in $E$. coli BL21(DE3). The purified protein displayed inhibitory activity against trypsin with $83 \%$ inhibition activity. Our study showed that metagenomics is a potential tool for discovery and exploitation of novel protease inhibitor from uncultivable microorganisms.

Acknowledgements: This work was supported by Vietnamese Ministry of Science and Technology under grant number: ĐTĐLCN.17/14.

\section{REFERENCES}

Agbowuro A. A., Huston W. A., Gamble A.B., Tyndall J. D. A., 2017. Proteases and protease inhibitors in infectious diseases. Med Res Rev., 38(4): 1295-1331.

Ashburner M., Ball C., Blake J., Botstein D., Butler H., Cherry J. M., Davis A. P., Dolinski K., Dwight S. S., Eppig J. T., Harris M. A., Hill D. P., Issel-Tarver L., Kasarskis A., Lewis S., Matese J. C., 
Richardson J. E., Ringwald M., Rubin G. M., Sherlock G., 2006. Gene ontology: tool for the unification of biology. Nat. Genet., 25(1): 25-29.

Baharum S. N., Beng E. K., Mokhtar M. A. A., 2010. Marine Microorganisms: Potential Application and Challenges. Journal of Biological Sciences., 10(6): 555-564.

Barrett A. J., Rawlings N. D., Woessner J. J. F., 1998. Hand book of proteolytic enzymes. Academic Press, London, pp. 1666.

Cantarel B. L., Coutinho P. M., Rancurel C., Bernard T., Lombard V., Henrissat B., 2009. The Carbohydrate-Active EnZymes database (CAZy): an expert resource for glycogenomics. Nucleic acids Res., 37(1): D233-D238.

Culligan E. P., Sleator R. D., Marchesi J. R., Hill C., 2014. Metagenomic Identification of a Novel Salt Tolerance Gene from the Human Gut Microbiome Which Encodes a Membrane Protein with Homology to a $b r p / b l h$-Family $\beta$-Carotene $15,15^{\prime}$ Monooxygenase. PLoS ONE., 9(7): e103318.

He R., Wang B., Wakimoto T., Wang M., Zhu L., Abe I., 2013. Cyclodipeptides from Metagenomic Library of a Japanese Marine Sponge. J. Braz. Chem. Soc., 24(12): 1926-1932.

Hyatt D., Chen G. L., LoCascio P. F., Land M. L., Larimer F. W., Hauser L. J., 2010. Prodigal: prokaryotic gen recognition and translation initiation site identification. BMC bioinformatics., 11: 119.

Jiang C. J., Hao Z. Y., Zeng R., Shen P. H., Li J. H., Wu B., 2011. Characterization of a Novel Serine Protease Inhibitor Gene from a Marine Metagenome. Marine. Drugs., 9(9): 1487-1501.
Karthik L., Kumar G., Keswani T., Bhattacharyya A., Chandar S. S., Rao K. V. B., 2014. Protease Inhibitors from Marine Actinobacteria as a Potential Source for Antimalarial Compound. PLoS ONE., 9(3): e90972.

Pimentel-Elardo S. M., Buback V., Gulder T. A. M., Bugni T. S., Reppart J., Bringmann G., Ireland C. M., Schirmeister T., Hentschel U., 2011. New Tetromycin Derivatives with Anti-Trypanosomal and Protease Inhibitory Activities. Marine. Drugs, 9(10): 1682-1697.

Ramadan A. U., Szesny M., Othman E. M., Schirmeister T., Grond S., Stopper H., Hentschel U., 2012. Antioxidant and AntiProtease Activities of Diazepinomicin from the Sponge-Associated Micromonospora Strain RV115. Marine. Drugs., 10(10): 2208-2221.

Sapna K., 2013. Isolation, purification, characterization and application of proteinaceous protease inhibitor from marine bacterium Pseudomonas mendocina BTMW 301. PhD Thesis, Cochin University of Science and Technology, Kerala, India, pp. 215.

Tabares P., Pimentel-Elardo S. M., Schirmeister T., Hünig T., Hentschel U., 2011. Anti-protease and immunomodulatory activities of bacteria associated with Caribbean sponges. Marine. Biotechnol., 13(5): 883-892.

Tatusov R. L., Natale D. A., Garkavtsev I. V., Tatusova T. A., Shankavaram U. T., Rao B. S., Kiryutin B., Galperin M. Y., Fedorova N. D., Koonin E. V., 2001. The COG database: new developments in phylogentic classification of proteins from complete genomes. Nucleic Acids Res., 29(1): 22-28.

Wahyudi A. T., Qatrunnada, Mubarik N.R., 2010. Screening and Characterization of Protease Inhibitors from Marine Bacteria 
Associated with Sponge Jaspis sp. HAYATI Journal of Biosciences., 17(4): 173-178.

Yin Y., Mao X., Yang J., Chen X., Mao F., $\mathrm{Xu}$ Y., 2012. dbCAN: a web resource for automated carbohydrate-active enzyme annotation. Nucleic Acids Res., 40(W1): W445-W451.

Zhu W., Lomsadze A., Borodovsky M., 2010. $\mathrm{Ab}$ initio gen identification in metagenomic sequence. Nucleic acids Res., 38(12): e132. 